\section{Advise Patients}

Workers with flavoring-related lung disease should be removed from further exposure and relocated within the facility to areas where they will not be exposed to diacetyl or similar flavoring chemicals.

Remind patients of the importance of:

- Using available exposure control methods such as ventilation hoods.

- Keeping flavoring containers tightly closed when not in use to prevent the contents from getting into the air

- Reading and understanding labels on containers and material safety data sheets for substances that they are exposed to in the workplace.

- Wearing personal protective equipment as appropriate.

Encourage patients to talk to their supervisors about:

- Training on hazard identification, procedures for safe handling of flavoring chemicals, and symptoms of flavoring-related lung disease.

- Exposure control methods (e.g., ventilation, isolation) and work practices (e.g., standard procedures for cleaning the workplace and for handling and storing flavoring chemicals).

- Wearing fit-tested respirators (protective breathing equipment) and other personal protective equipment (e.g., gloves, eye protection, aprons) to reduce exposures.

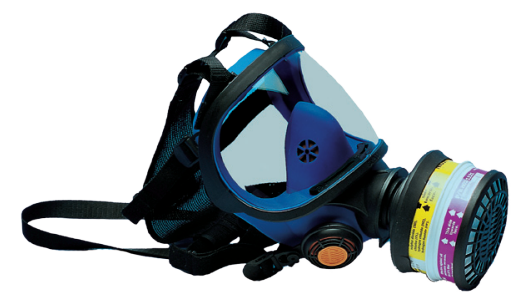

In addition to exposure control methods, use a NIOSHcertified full-facepiece air-purifying respirator with at a minimum NIOSH-certified organic vapor cartridges and particulate cartridges/filters and other cartridges as necessary.

\section{Report}

Much remains to be learned about the nature of this serious lung disease. You can play an important role. With your assistance, the information gained from reporting can help identify high-risk work settings and guide efforts to prevent additional cases of flavoring-related lung disease.

\section{Reporting of Cases}

Please report any cases of occupational lung disease with or without fixed airways obstruction that you suspect are attributable to flavoring chemicals to your State Health Department and the NIOSH Division of Respiratory Disease Studies (Telephone: 1-800-232-2114).

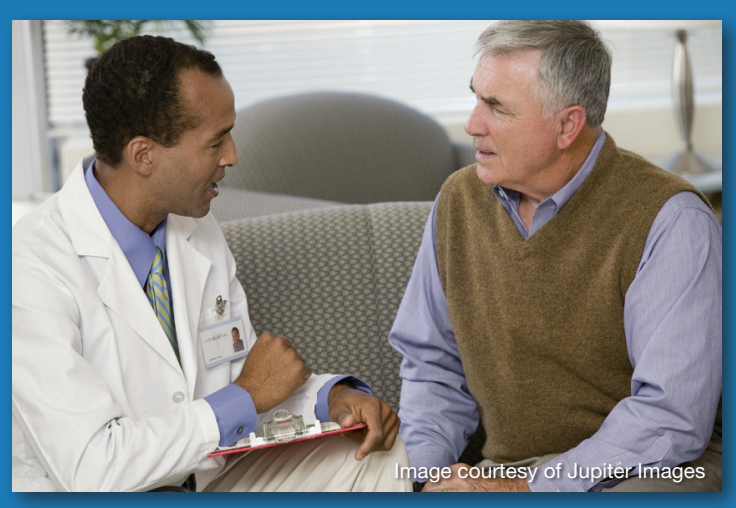

\section{Additional Information}

To learn more about the effects of flavorings:

\section{NIOSH Web site:}

www.cdc.gov/niosh/topics/flavorings

NIOSH [2003]. NIOSH Alert: Preventing Lung Disease in Workers Who Use or Make Flavorings [http://www.cdc.gov/niosh/docs/2004-110].

\section{NIOSH}

Telephone: 1-800-CDC-INFO (1-800-232-4636) TTY: 1-888-232-6348 | E-mail: cdcinfo@cdc.gov or visit the NIOSH Web site at www.cdc.gov/niosh

For a monthly update on news at NIOSH, subscribe to NIOSH eNews by visiting www.cdc.gov/niosh/eNews

DHHS (NIOSH) Publication No. 2012-148 (supersedes 2012-107)

May 2012

SAFER • HEALTHIER ・ PEOPLETM

DEPARTMENT OF HEALTH AND HUMAN SERVICES Centers for Disease Control and Prevention

National Institute for Occupational Safety and Health $\checkmark$ Recognize $\checkmark$ Respond $\checkmark$ Report

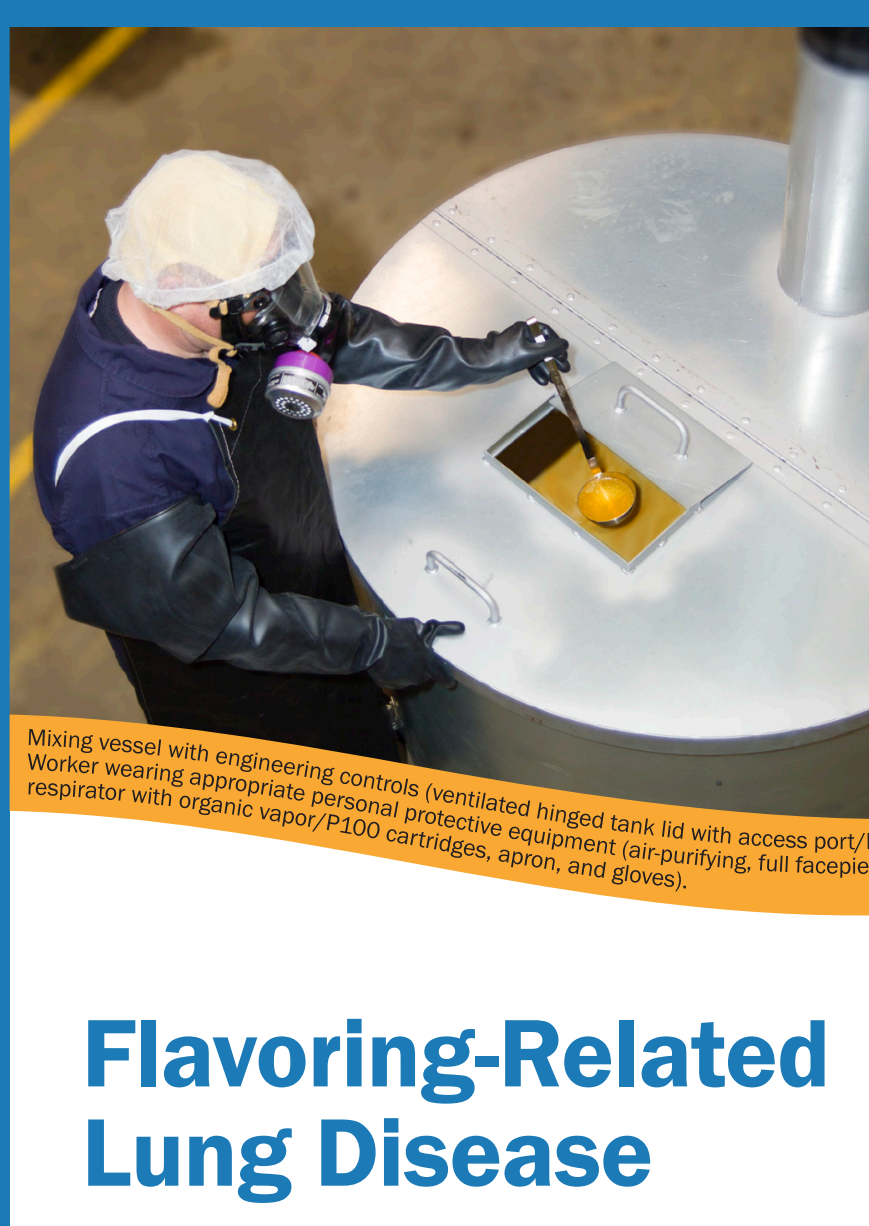

Information for Healthcare Providers

Workers who breathe flavoring chemicals containing diacetyl, such as butter flavoring, may be at risk of severe obstructive lung disease. 


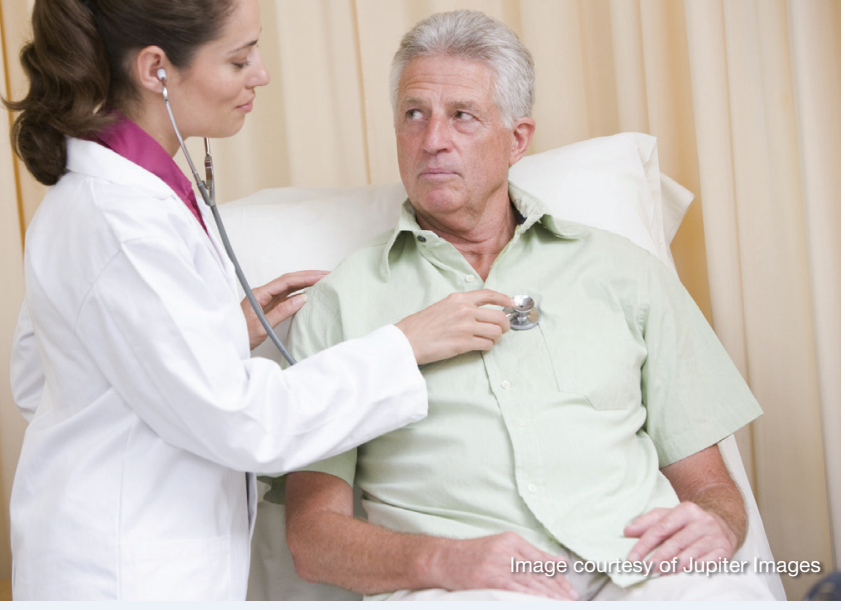

\section{What is the problem/illness?}

Severe obstructive lung disease has been identified in people who work with flavoring chemicals containing diacetyl, including butter flavoring. Animal studies suggest that some flavoring chemicals similar to diacetyl, such as 2,3-pentanedione, may also be a hazard. Cases of severe lung disease have occurred in microwave popcorn plants and flavoring production plants. Lung biopsies in some affected people have shown the presence of a rare condition; constrictive bronchiolitis obliterans (also referred to as constrictive bronchiolitis, obliterative bronchiolitis, and bronchiolitis obliterans). In some workers, fixed airways obstruction has been severe enough to be fatal.

\section{Who is at risk?}

Workers who make, use, or work near flavoring chemicals containing diacetyl or 2,3-pentanedione may be at risk. Settings where these exposures may occur include:

- Flavoring plants

- Microwave popcorn plants

- Commercial and retail bakeries

- Snack food and candy plants

- Dairy products and packaged vegetable oil plants (e.g., margarine, cooking oil)

- Other flavored food production plants

Exposures in the flavoring industry and in microwave popcorn production have caused workers to have severe lung disease. The burden of hazardous exposures and risk to workers in other settings remains unclear. However, it is important to consider the possibility of flavoring-related lung disease in workers who have been exposed to diacetyl or similar flavoring chemicals and have respiratory symptoms.

\section{Recognize}

\section{Take an Occupational History}

Take an occupational history to avoid missing exposures to flavorings. Affected workers may have used flavoring chemicals for a few months to a period of years. Ask your patients about jobs held, exposure to flavoring chemicals (and other dusts, mists, gases, vapors, or fumes), and if other coworkers have experienced similar symptoms. Sometimes workers are initially misdiagnosed with asthma, chronic bronchitis, emphysema, or pneumonia; or their symptoms are attributed to smoking.

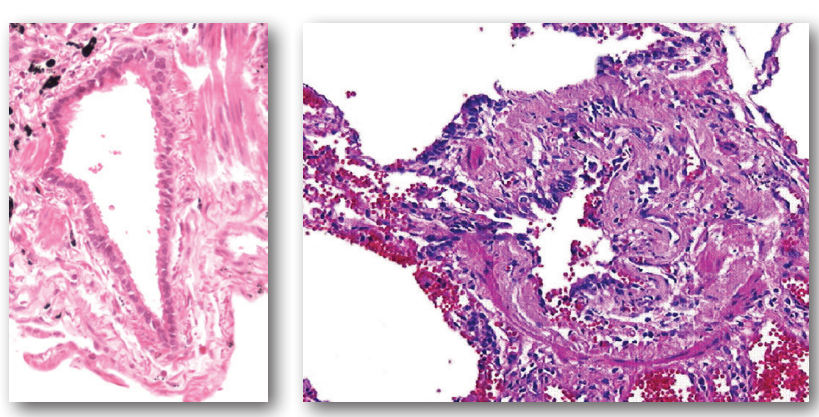

Healthy bronchiole (left) and bronchiole of an affected worker (right) showing patterns of constrictive bronchiolitis obliterans.

\section{Ask About Symptoms}

Symptoms are not present in all cases. When present, symptoms can range in severity. The main respiratory symptoms experienced by workers include:

- Cough (usually without phlegm)

- Shortness of breath on exertion

- Wheezing

Other symptoms experienced by some workers include:

- Fever, night sweats, or weight loss

- Frequent or persistent eye, nose, throat, or skin irritation

In cases of flavoring-related lung disease, respiratory symptoms do not typically improve when the worker goes home at the end of the workday, on weekends, or on vacations. The symptoms often have a gradual onset but can occur suddenly. Work-related exposure to butter flavoring chemicals might also lead to asthma or exacerbate pre-existing asthma.

\section{Respond}

\section{Conduct a Medical Evaluation}

Medical testing may include the following:

- Spirometry most often shows an obstructive pattern of ventilatory impairment that does not improve after bronchodilators and sometimes shows a restrictive or mixed obstructive/restrictive pattern of ventilator impairment. Careful comparison of spirometry test results over time is important to identify potentially concerning declines in lung function. The rapid decline of lung function (even if it is still within the normal range) or the finding of a reduced forced expiratory volume in 1 second (FEV1) should trigger additional evaluation.

- Lung volume assessments are consistent with hyperinflation

- Carbon monoxide diffusing capacity measurement is generally normal, especially early in the disease.

- Chest $\mathbf{x}$-rays are usually normal but may show hyperinflation.

- High-resolution computerized tomography scans of the chest at full inspiration and expiration often reveal heterogeneous air trapping on the expiratory view and may demonstrate mosaic attenuation, bronchial wall thickening, cylindric bronchiectasis, and/or scattered ground glass opacities.

- Lung biopsies are not generally required for diagnosis, but may reveal evidence of constrictive bronchiolitis obliterans. Sometimes the term clinical bronchiolitis obliterans is used for patients who have medical test findings consistent with the disease constrictive bronchiolitis obliterans but do not have confirmatory biopsy results.

\section{Perform Medical Management}

Patients with flavoring-related lung disease should be removed from further exposure to diacetyl or similar flavoring chemicals. Removal from exposure does not usually result in significant improvement, but the level of impairment often stabilizes. No specific treatment has been shown to reverse abnormalities in lung function. Symptomatic therapy should be provided as appropriate. 